\title{
Optimal Insurance under Heterogeneous Belief
}

\author{
Huimin Yu, Ying Fang \\ School of Mathematics and Statistics, Shandong Normal University, Jinan, China \\ Email: 1909385517@qq.com, fangying319@163.com
}

How to cite this paper: Yu, H. M., \& Fang, Y. (2020). Optimal Insurance under Heterogeneous Belief. Journal of Financial Risk Management, 9, 179-189.

https://doi.org/10.4236/jfrm.2020.93010

Received: June 23, 2020

Accepted: July 31, 2020

Published: August 3, 2020

Copyright $\odot 2020$ by author(s) and Scientific Research Publishing Inc. This work is licensed under the Creative Commons Attribution International License (CC BY 4.0).

http://creativecommons.org/licenses/by/4.0/

\begin{abstract}
In this paper, we study an optimal insurance problem, which allows the insured and the insurer to have heterogeneous probability beliefs in the distribution of potential losses, on the basis of which we maximize the expected utility of the insured's final wealth. In order to reduce ex-post moral hazard, we assume that the alternative insurance contract follows the principle of indemnity and incentive compatibility constraints. Under the assumption of Wang's premium principle, we derive a necessary and sufficient condition for the optimal solution. Then we discuss some particular characteristics of the optimal solution and the optimality of no insurance and full insurance.
\end{abstract}

\section{Keywords}

Optimal Insurance, Heterogeneous Belief, Incentive Compatibility, Wang's Premium Principle

\section{Introduction}

The research on insurance began in 1960s. Since the seminal work of (Arrow, 1963), the optimal insurance strategy has aroused great interest in research and practice, and has laid significant foundation in insurance economics. (Arrow, 1963) first studied this issue from the perspective of the insured, who seeks to maximize the expected utility of the final wealth. Assuming the insurance premium is calculated according to the expected value principle, the author showed that the stop loss treaty is the optimal, and this conclusion is known as Arrow's deductible theorem.

In recent years, with the rapid development of insurance industry and the improvement of economic level, people's insurance awareness is gradually improving, and more and more people have accepted insurance. When facing huge risks, the insured can choose to share the risk with the insurance company by purchasing the insurance contract. In the study of optimal insurance, optimal 
forms of insurance depend on premium principles and optimization criteria. There are many different premium principles and optimization criteria to choose from, which stimulate different research flows. For example, (Young, 1999) studied the relatively complicated principle of Wang's premium. In the past decade, one optimization criteria commonly used in the study of optimal insurance is minimizing the risk measure of a company's risk, for example, (Cai \& Tan, 2007), (Chi \& Lin, 2014), (Cheung, 2010), and references therein, studied the optimal insurance or reinsurance under the value-at-risk (VaR) and the conditional tail expectation (CTE) risk measures. In addition, some literatures studied the optimal insurance by maximizing the non-expected utility preference, for example, (Xu, Zhou, \& Zhuang, 2019) established the optimal insurance model when the preference of the insured is rank dependent utility type.

It should be noted that in these studies, both parties of the insurance contract have the same probability belief on potential loss distribution. However, this assumption has been questioned in insurance economics. In fact, information asymmetry is also universal existence in the insurance practice. (Gollier, 2013) pointed out that even if information is symmetrical, people still have differences in the distribution of risk. And before that, (Savage, 1972) mainly showed that the insured and the insurer will make decisions according to their own views and emphasized that the insured and the insurer usually make decisions based on personal probability view, so it is normal for them to have different views on the distribution of potential losses.

As far as we know, (Marshall, 1992) was the first to study the optimal insurance problem with belief heterogeneity, and obtained some interesting results. The author proved that the optimal insurance contract with nonnegative indemnity constraint can have any form of expression through heuristic analysis, and then introduced a very special form of belief heterogeneity. This particularity is mainly reflected in the case of non-zero loss, both parties of the contract have the same conditional distribution, and the probability quality of zero loss of the insured is less than that of the insurer. However, this is a rather restrictive approach to belief heterogeneity, which does not have universality. In addition, the optimal solution does not follow the principle of indemnity, that is, indemnity must be nonnegative and less than the loss itself.

Then (Gollier, 2013) considered another new form of heterogeneous belief, assuming that the insured is more optimistic about the insurable loss than the insurer in the sense of monotone likelihood ratio (MLR). Although the expression of the optimal solution was not derived, the author proved that the optimal marginal indemnity should be less than 1 and can be negative. On the premise that belief heterogeneity is compatible and the insurance contract satisfies the principle of indemnity, (Ghossoub, 2016) and (Ghossoub, 2017) obtained the optimality of variable deductible insurance. The author proved that when the compensation of the optimal solution increases in the loss, the optimal marginal compensation can be strictly greater than 1 . According to the research of 
(Huberman, Mayers, \& Smith Jr., 1983), when the marginal compensation is negative or strictly greater than 1 , the optimal insurance policy will lead toex post moral hazard. (Jiang, Ren, Yang, \& Hong, 2019) studied the optimal reinsurance problem of cooperative game under the heterogeneous belief and mainly established a Pareto optimal model to give the most beneficial insurance policy for both parties. But a similar problem appeared, that is, the optimal insurance policy did not ex-post moral hazard. Therefore, in order to solve this problem, (Huberman, Mayers, \& Smith Jr., 1983) suggested that the alternative insurance contract should meet the incentive compatibility condition in addition to the indemnity principle. In other words, the insurer and the insured are required to pay more for the insurable loss. This is equivalent to that marginal indemnity should be non-negative and less than 1. For details, please refer to literature (Liu, Zhao, Liu, \& Chen, 2017). In addition, (Chi, 2019) gave the optimality of stop loss insurance under belief heterogeneity. In the MHR sense, when the insurer is more optimistic about the conditional distribution of non-zero loss than the insured, they obtained the optimality of the deductible insurance. By comparing (Chi, 2019) and (Ghossoub, 2017), we find that applying incentive compatibility constraint can change from variable deductible to stop loss insurance contract.

It should be noted here that the above research on belief heterogeneity is limited to some special forms. Recently, (Chi \& Zhuang, 2020) studied optimal insurance according to the principle of the expected value under the heterogeneous belief, explored some properties of the optimal solution, and obtained the optimal strategy for the special form of heterogeneous belief. However, the optimal insurance problem with belief heterogeneity and incentive compatibility is far from fully solved. Based on this paper, we extend the expected value premium principle to the more complex Wang's premium principle and establish some new conclusions.

The rest of the paper is organized as follows. In the second section, we introduce an optimal insurance model with heterogeneous belief, in which the insurance contract satisfies the principle of indemnity and incentive compatibility constraints, and prove the existence and uniqueness of the optimal solution. In the third section, we establish a sufficient and necessary condition of the optimal solution. Then, we further explore some properties of the optimal solution according to this condition. In addition, we discuss the optimality of no insurance and full insurance in detail, and give the specific numerical analysis. In the fourth section, we give a summary description and point out the follow-up research directions and problems.

\section{Model}

Suppose for a fixed time period, let $\Omega$ be the set of states, $W_{0}$ be the initial wealth of the insured, $X$ be the random loss faced by the insured, where $X$ is a nonnegative bounded random variable defined in probability space $(\Omega, \mathcal{F}, P)$ with $\mathrm{E}^{P}(X)>0$ and $P\left(X<W_{0}\right)=1$. Denote by $\mathcal{F}$ the sigma algebra gen- 
erated by $X$, and by $P$ the subjective probability measure of the insured. In order to reduce the damage caused by huge claims, the insured usually purchase insurance to share part of the risk. We suppose that $f(X)$ is the ceded loss of the insured, then the retained loss is $R_{f}(X)=X-f(X)$, where $f(x)$ is the so-called ceded loss function.

The principle of indemnity is a widely accepted principle in insurance, which requires that the ceded loss function is nonnegative and less than the loss itself. Mathematically, we describe it as $0 \leq f(x) \leq(x)$. However, (Huberman, Mayers, \& Smith Jr., 1983) pointed out that ex-post moral hazard may be caused in this case. Therefore, in order to eliminate ex-post moral hazard, we assume that the ceded loss function must satisfy the incentive compatibility condition, that is to say, both the ceded loss function and the retained loss function are increasing functions, which is further equivalent to $0 \leq f^{\prime}(x) \leq 1$ almost everywhere, where $f^{\prime}(x)$ is the derivative of $f(x)$. In this article, we follow the method of (Huberman, Mayers, \& Smith Jr., 1983) to study the optimal ceded loss function in set

$$
\mathbb{C}:=\left\{0 \leq f(x) \leq x: 0 \leq f^{\prime}(x) \leq 1\right\} .
$$

In this paper, we assume that the insurance premium is calculated by Wang's premium principle. For any nonnegative random variable $Z$, there are

$$
\pi(Z)=\int_{0}^{\infty} g(Q(Z>z)) \mathrm{d} z=\mathrm{E}_{g}^{Q}(Z),
$$

where $\pi(\cdot)$ is the principle of insurance premium, $Q$ is the subjective probability measure of the insurer defined in $(\Omega, \mathcal{F}), g(\cdot)$ is a nondecreasing distortion function satisfying $g(0)=0, g(1)=1$. But it should be noted that $Q$ may not equal to $P$. This model reflects the different probability beliefs about potential loss between insured and insurer.

In the case of the insurance contract with $f(X)$, we make $W_{f}(X)$ represent the insured's final wealth, we have

$$
W_{f}(X)=W_{0}-X+f(X)-\mathrm{E}_{g}^{Q}(f(X)) .
$$

In this paper, we further assume that the insured is risk averse. According to (Arrow, 1963), we use the expected utility theory to describe the insured's preference, that is, the insured wants to maximize the expected utility of its final wealth. Then, our optimal insurance problem is described as

$$
\max _{f(X) \in \mathbb{C}} \mathrm{E}^{P}\left[U\left(W_{f}(X)\right)\right]
$$

where $U(\cdot)$ is the utility function of the insured and satisfies $U^{\prime}(\cdot)>0, U^{\prime \prime}(\cdot)<0$.

Before solving the problem, we prove the existence and uniqueness of the optimal solution of Problem (2.1) by using the similar method in (Chi \& Zhuang, 2020).

Lemma 2.1 (1) There are solutions to Problem (2.1).

(2) If 0 belongs to the support of $X$ under probability measure $P$, that is to say, there is $P(X>\varepsilon)>0$ for any $\varepsilon>0$, then the Problem (2.1) has a unique op- 
timal solution.

In order to prove lemma 2.1, we give a well-known Arzelà-Ascoli theorem. Before stating it, we shall introduce the definitions of uniform boundedness and equicontinuity.

Definition 2.1. A sequence of real valued continuous functions $\left\{f_{n}\right\}_{n \in N}$ defined on an interval $[a, b]$ is uniformly bounded if there is a number $B$ such that $\left|f_{n}(x)\right| \leq B$ for every $n$ and $x \in[a, b]$.

Definition 2.2. A sequence of real valued continuous functions $\left\{f_{n}\right\}_{n \in N}$ is said to be equicontinuous, if, for every $\varepsilon>0$ and $x$, there exists a $\delta>0$ such that $\left|f_{n}(x)-f_{n}(y)\right|<\varepsilon$ whenever $|x-y|<\delta$ for all functions $f_{n}$.

It's worth noting that, for any sequence of ceded loss functions from the admissible set $\mathbb{C}$, it is easy to see that this sequence is uniformly bounded and equicontinuous. Next, we will introduce this important theorem, see Theorem 2.3 .

Theorem 2.3 (Arzelà-Ascoli theorem) Consider a sequence of realvalued continuous functions $\left\{f_{n}\right\}_{n \in N}$ real valued continuous functions $\left\{f_{n}\right\}_{n \in N}$ defined on a closed and bounded interval $[a, b]$ of the real line. If this sequence is uniformly bounded and equicontinuous, then there exists a subsequence $\left\{f_{n_{k}}\right\}$ that converges uniformly to a continuous function on $[a, b]$.

Then, we continue to prove lemma 2.1.

Proof. Since $X$ is a nonnegative bounded random variable, we suppose $X \in[0, M]$. Note that the ceded loss function belongs to set $\mathbb{C}$ and satisfies Lipschitz continuity, then $\left\{f_{n}\right\}_{n \geq 1}$ must be uniformly bounded and equicontinuous. Then according to Theorem 2.3, there must be a sequence $\left\{f_{n_{k}}\right\}_{k \geq 1}$ uniformly converging to $\left\{f_{n}\right\}$. Therefore, the optimal solution of Problem (2.1) exists.

Next, we prove the uniqueness of the optimal solution. First, suppose $f_{1}$ and $f_{2}$ are the optimal solutions of the Problem (2.1), then

$$
m:=\mathrm{E}^{P}\left[U\left(W_{f_{1}}(X)\right)\right]=\mathrm{E}^{P}\left[U\left(W_{f_{2}}(X)\right)\right] .
$$

Define $f_{p}(X)=p f_{1}(X)+(1-p) f_{2}(X)$ for any $p \in[0,1]$. It's easy to get $f_{p}(X) \in \mathbb{C}$ and $m \geq \mathrm{E}^{P}\left[U\left(W_{f_{p}}(X)\right)\right]$, then according to the concavity of $U(\cdot)$, we can get

$$
\mathrm{E}^{P}\left[U\left(W_{f_{p}}(X)\right)\right] \geq p \mathrm{E}^{P}\left[U\left(W_{f_{1}}(X)\right)\right]+(1-p) \mathrm{E}^{P}\left[U\left(W_{f_{2}}(X)\right)\right]=m .
$$

Therefore, we have

$$
\mathrm{E}^{P}\left[U\left(W_{f_{1}}(X)\right)\right]=\mathrm{E}^{P}\left[U\left(W_{f_{2}}(X)\right)\right]=\mathrm{E}^{P}\left[U\left(W_{f_{p}}(X)\right)\right]=m,
$$

and because of inequality $U^{\prime \prime}(\cdot) \leq 0$, we can get $W_{f_{p}}(X)=W_{f_{2}}(X)=W_{f_{1}}(X)$ almost surely under $P$. Note that $f_{i}(0)=0, i=1,2$, If 0 belongs to the support of $X$ under probability measure $P$, then when $X \rightarrow 0$ has $\mathrm{E}_{g}^{Q}\left(f_{1}(X)\right)=\mathrm{E}_{g}^{Q}\left(f_{2}(X)\right)$, it means that $f_{1}(X)=f_{2}(X)$, that is $P\left(f_{1}(X)=f_{2}(X)\right)=1$. So the unique- 
ness is proved.

Remark 2.4. It should be noted that the probability of zero loss in practice is usually positive. For details, see (Smith, 1968). It is worth noting that the main difference between the result of ours and (Chi \& Zhuang, 2020) is that $\pi(\cdot)$ does not satisfy the additivity under Wang's premium principle, so even if $Q$ is absolutely continuous with respect to $P$, we cannot derive the uniqueness of the optimal solution.

\section{Main Results}

Next, we establish a necessary and sufficient condition for the ceded loss function to be the optimal solution in the following theorem.

Theorem 3.1. The ceded loss function $f^{*}(t)$ is the optimal solution of the Problem (2.1) if and only if it satisfies

$$
f^{* \prime}(t)=\left\{\begin{array}{l}
1, L(t)>0 \\
k(t), L(t)=0 \\
0, L(t)<0
\end{array}\right.
$$

where $k(t)$ is bounded almost everywhere in $[0,1], L(t)$ is defined as

$$
L(t):=\frac{\mathrm{E}^{P}\left[U^{\prime}\left(W_{f^{*}}(X)\right) \cdot \mathrm{I}_{\{X>t\}}\right]}{\mathrm{E}^{P}\left[U^{\prime}\left(W_{f^{*}}(X)\right)\right]}-\frac{\mathrm{E}^{P}\left[U^{\prime}\left(W_{f^{*}}(X)\right) \cdot \mathrm{E}_{g}^{Q}\left(\mathrm{I}_{\{X>t\}}\right)\right]}{\mathrm{E}^{P}\left[U^{\prime}\left(W_{f^{*}}(X)\right)\right]} .
$$

for any $t \in[0, \infty]$, here $\mathrm{I}_{A}$ represents an indicator function of event $A$.

Proof. We first assume that $f^{*}(t)$ is the optimal solution of the Problem (2.1). For any insurance contract $f(t)$, we define

$$
f_{\theta}(t):=(1-\theta) f^{*}(t)+\theta f(t) \text { and } \Phi(\theta)=\mathrm{E}^{P}\left[U\left(W_{f_{\theta}}(X)\right)\right] \text {. }
$$

It is obvious that we have $f_{\theta}(t) \in \mathbb{C}$ and $\Phi(\theta)$ is concave with respect to $\theta$. Then according to the concavity of $U(\cdot)$ and the optimality of $f^{*}(t)$, we have

$\frac{\Phi(\theta)-\Phi(0)}{\theta}=\frac{\mathrm{E}^{P}\left[U\left(W_{f_{\theta}}(X)\right)\right]-\mathrm{E}^{P}\left[U\left(W_{f^{*}}(X)\right)\right]}{\theta}$

$=\mathrm{E}^{P}\left[U^{\prime}\left(W_{f_{\theta}}(X)\right) \cdot\left\{f(X)-f^{*}(X)-\mathrm{E}_{g}^{Q}\left(f(X)-f^{*}(X)\right)\right\}\right]$

$\stackrel{\theta \downarrow_{0}}{\longrightarrow} \mathrm{E}^{P}\left\{U^{\prime}\left(W_{f^{*}}(X)\right) \cdot\left[f(X)-f^{*}(X)-\mathrm{E}_{g}^{Q}\left(f(X)-f^{*}(X)\right)\right]\right\}$

$=\mathrm{E}^{P}\left\{U^{\prime}\left(W_{f^{*}}(X)\right) \cdot\left[\int_{0}^{\infty} \mathrm{I}_{\{X>t\}}\left(f^{\prime}(t)-f^{* \prime}(t)\right) \mathrm{d} t-\mathrm{E}_{g}^{Q}\left(\int_{0}^{\infty} \mathrm{I}_{\{X>t\}}\left(f^{\prime}(t)-f^{* \prime}(t)\right) \mathrm{d} t\right)\right]\right\}$

$=\mathrm{E}^{P}\left[U^{\prime}\left(W_{f^{*}}(X)\right)\right] \cdot \int_{0}^{\infty} L(t)\left(f^{\prime}(t)-f^{* \prime}(t)\right) \mathrm{d} t \leq 0$

where $L(t)$ is defined in(3.2), the third equation is based on

$f(x)=\int_{0}^{\infty} \mathrm{I}_{\{x>t\}} f^{\prime}(t) \mathrm{d} t$ for any $x \geq 0$. It is noted that $\frac{\Phi(\theta)-\Phi(0)}{\theta} \leq 0$ is true, so the above inequality holds for any ceded loss function $f(X) \in \mathbb{C}$. After 
verification, $f^{* \prime}(t)$ meets (3.1).

Conversely, if the ceded loss function $f^{* \prime}(t)$ satisfies (3.1), then for any $f(X) \in \mathbb{C}$, there are

$$
\begin{aligned}
& \mathrm{E}^{P}\left[U^{\prime}\left(W_{f^{*}}(X)\right)\right]-\mathrm{E}^{P}\left[U^{\prime}\left(W_{f}(X)\right)\right] \\
& \geq \mathrm{E}^{P}\left\{U^{\prime}\left(W_{f^{*}}(X)\right) \cdot\left[f^{*}(X)-f(X)-\mathrm{E}_{g}^{Q}\left(f^{*}(X)\right)+\mathrm{E}_{g}^{Q}(f(X))\right]\right\} \\
& =\mathrm{E}^{P}\left[U^{\prime}\left(W_{f^{*}}(X)\right)\right] \cdot \int_{0}^{\infty} L(t) \cdot\left(f^{* \prime}(t)-f^{\prime}(t)\right) \mathrm{d} t \geq 0
\end{aligned}
$$

Thus, $f^{*}(t)$ is the optimal solution of the Problem (2.1).

Theorem 3.1 gives the general form of the optimal solution of Problem (2.1). Specifically, the marginal indemnity of $f^{* \prime}(t)$ should be 0 or 1 , and there are some irregularities at the critical point of $L(t)=0$. Moreover, Theorem 3.1 can be used to identify the optimality of any given acceptable ceded loss function. So as to further explore the properties of the optimal solution.

\subsection{Properties of the Optimal Solution}

First, we introduce $M_{P}$ and $M_{Q}$, which are the essential supremum of $X$ under $P$ and $Q$.

The mathematical expression can be expressed as follows:

$$
\begin{aligned}
& M_{P}(x):=\inf \{x: P(X \leq x)=1\}, \\
& M_{Q}(x):=\inf \{x: Q(X \leq x)=1\} .
\end{aligned}
$$

Proposition 3.1. We have $f^{* \prime}(t)=0$, when any $t \geq 0$ satisfies $\mathrm{E}_{g}^{Q}\left[\mathrm{I}_{\{X>t\}}\right]>1$. Further, when $M_{P}<M_{Q}$ for any $t \in\left[M_{P}, M_{Q}\right)$, there is $f^{* \prime}(t)=0$.

Proof. When $\mathrm{E}_{g}^{Q}\left[\mathrm{I}_{\{X>t\}}\right]>1$, we have

$$
\mathrm{E}^{P}\left[U^{\prime}\left(W_{f^{*}}(X)\right) \cdot \mathrm{E}_{g}^{Q}\left(\mathrm{I}_{\{X>t\}}\right)\right]>\mathrm{E}^{P}\left[U^{\prime}\left(W_{f^{*}}(X)\right)\right],
$$

this shows that

$$
L(t)=\frac{\mathrm{E}^{P}\left[U^{\prime}\left(W_{f^{*}}(X)\right) \cdot \mathrm{I}_{\{X>t\}}\right]}{\mathrm{E}^{P}\left[U^{\prime}\left(W_{f^{*}}(X)\right)\right]}-\frac{\mathrm{E}^{P}\left[U^{\prime}\left(W_{f^{*}}(X)\right) \cdot \mathrm{E}_{g}^{Q}\left(\mathrm{I}_{\{X>t\}}\right)\right]}{\mathrm{E}^{P}\left[U^{\prime}\left(W_{f^{*}}(X)\right)\right]}<0 .
$$

Therefore, according to Theorem 3.1, we have $f^{* \prime}(t)=0$ for any $t \geq 0$ satisfies $\mathrm{E}_{g}^{Q}\left[\mathrm{I}_{\{X>t\}}\right]>1$.

Moreover, if $M_{P}<M_{Q}$ for any $t \in\left[M_{P}, M_{Q}\right)$, we have $P(X>t)=0$, then $L(t)$ can be rewritten as

$$
L(t)=-\frac{\mathrm{E}^{P}\left[U^{\prime}\left(W_{f^{*}}(X)\right) \cdot \mathrm{E}_{g}^{Q}\left(\mathrm{I}_{\{X>t\}}\right)\right]}{\mathrm{E}^{P}\left[U^{\prime}\left(W_{f^{*}}(X)\right)\right]}<0 .
$$


So we have $f^{* \prime}(t)=0$ for any $t \in\left[M_{P}, M_{Q}\right)$.

The above proposition gives the point where the optimal marginal indemnity is 0 . Then according to the similar method, we discuss the point where the optimal marginal indemnity is 1 . See the following proposition.

Proposition 3.2. If $P(X>t)-\frac{\mathrm{E}^{P}\left[U^{\prime}\left(W_{f^{*}}(X)\right) \cdot \mathrm{E}_{g}^{Q}\left(\mathrm{I}_{\{X>t\}}\right)\right]}{\mathrm{E}^{P}\left[U^{\prime}\left(W_{f^{*}}(X)\right)\right]}>0$ for any $t \geq 0$, then $f^{* \prime}(t)=1$. In particular, when the distortion function $g(x)=x$ and $M_{Q}<M_{P}$, there is $f^{* \prime}(t)=1$.

Proof. According to $U^{\prime \prime}(\cdot)<0$, we can get that $U^{\prime}\left(W_{f^{*}}(X)\right)$ is comonotonic with $\mathrm{I}_{\{X>t\}}$, Which shows

$$
\begin{aligned}
L(t) & =\frac{\mathrm{E}^{P}\left[U^{\prime}\left(W_{f^{*}}(X)\right) \mathrm{I}_{\{X>t\}}\right]}{\mathrm{E}^{P}\left[U^{\prime}\left(W_{f^{*}}(X)\right)\right]}-\frac{\mathrm{E}^{P}\left[U^{\prime}\left(W_{f^{*}}(X)\right) \cdot \mathrm{E}_{g}^{Q}\left(\mathrm{I}_{\{X>t\}}\right)\right]}{\mathrm{E}^{P}\left[U^{\prime}\left(W_{f^{*}}(X)\right)\right]} \\
& \geq P(X>t)-\frac{\mathrm{E}^{P}\left[U^{\prime}\left(W_{f^{*}}(X)\right) \cdot \mathrm{E}_{g}^{Q}\left(\mathrm{I}_{\{X>t\}}\right)\right]}{\mathrm{E}^{P}\left[U^{\prime}\left(W_{f^{*}}(X)\right)\right]}>0
\end{aligned}
$$

Therefore, we have $f^{* \prime}(t)=1$ for any $t \geq 0$ because of Theorem 3.1.

On the other hand, if $g(x)=x$, then $\mathrm{E}_{g}^{Q}\left(\mathrm{I}_{\{X>t\}}\right)=\mathrm{E}^{Q}\left[\mathrm{I}_{\{X>t\}}\right]=Q(X>t)$ for any $t \in\left[M_{Q}, M_{P}\right)$, that is to say $Q(X>t)=0$, which in turn implies

$$
L(t) \geq P(X>t)-\frac{\mathrm{E}^{P}\left[U^{\prime}\left(W_{f^{*}}(X)\right) \cdot Q(X>t)\right]}{\mathrm{E}^{P}\left[U^{\prime}\left(W_{f^{*}}(X)\right)\right]}=P(X>t)>0 .
$$

Therefore, we have $f^{* \prime}(t)=1$.

In this case, the conclusion is consistent with (Chi \& Zhuang, 2020), who uses the expected value principle to establish the condition that the optimal marginal indemnity is 1 when the safety loading coefficient exists.

\subsection{Optimality of Full Insurance and No Insurance}

The optimality of full insurance and no insurance has always been a hot topic in the research of demand theory in insurance economics. In this section, we will use Theorem 3.1 to discuss their optimality.

Proposition 3.3. Full insurance is an optimal solution to Problem (2.1) if and only if

$$
P(X>t)>\mathrm{E}^{P}\left[\mathrm{E}_{g}^{Q}\left(\mathrm{I}_{\{X>t\}}\right)\right]
$$

holds for any $t \geq 0$.

Proof. According to Theorem 3.1, full insurance is an optimal solution if and only if $L(t) \geq 0$, where $L(t)$ can be described as 


$$
\begin{aligned}
L(t) & =\frac{\mathrm{E}^{P}\left[U^{\prime}(W-\pi) \cdot \mathrm{I}_{\{X>t\}}\right]}{\mathrm{E}^{P}\left[U^{\prime}(W-\pi)\right]}-\frac{\mathrm{E}^{P}\left[U^{\prime}(W-\pi) \cdot \mathrm{E}_{g}^{Q}\left(\mathrm{I}_{\{X>t\}}\right)\right]}{\mathrm{E}^{P}\left[U^{\prime}(W-\pi)\right]} \\
& =P(X>t)-E^{P}\left[\mathrm{E}_{g}^{Q}\left(\mathrm{I}_{\{X>t\}}\right)\right]
\end{aligned}
$$

Obviously, this conclusion has been proved to be complete.

Proposition 3.4. No insurance is an optimal solution to Problem (2.1) if and only if

$$
\mathrm{E}^{P}\left[U^{\prime}(W-X) \mathrm{I}_{\{X>t\}}\right] \leq \mathrm{E}^{P}\left[U^{\prime}(W-X) \cdot \mathrm{E}_{g}^{Q}\left(\mathrm{I}_{\{X>t\}}\right)\right] .
$$

Proof. According to Theorem 3.1, no insurance is the optimal solution of Problem (2.1) if and only if $L(t) \leq 0$. So the conclusion is proved.

Next, we use a specific example to further illustrate the case that no insurance is optimal.

Example 3.5. In this example, suppose that random loss $X$ follows a truncated exponential distribution under probability measure $P$, and the probability density function is

$$
f(x)=\frac{\mathrm{e}^{-x}}{1-\mathrm{e}^{-20}} I_{\{x \leq 20\}}, \quad \forall x \geq 0 .
$$

We let $W_{0}=20, U(s)=-\mathrm{e}^{-s}, Q\{X>t\}=18 \cdot P\{X>t\}$, distortion function $g(x)=x$.

After a series of calculations, we have

$$
\frac{\mathrm{E}^{P}\left[U^{\prime}\left(W_{0}-X\right) \cdot \mathrm{I}_{\{X>10\}}\right]}{\mathrm{E}^{P}\left[U^{\prime}\left(W_{0}-X\right)\right]}=\frac{1}{2} .
$$

And because $g(x)=x$, then $\mathrm{E}_{g}^{Q}\left[\mathrm{I}_{\{X>t\}}\right]=Q\{X>t\}$. After calculation, we have $Q\{X>t\}>\frac{1}{2}$, and then we deduce $L(t) \leq 0$, that is to say, in this case, no insurance is optimal.

\section{Concluding}

In conclusion, this paper analyzes an optimal insurance design problem, which not only satisfies the incentive compatible conditions according to the requirements of (Huberman, Mayers, \& Smith Jr., 1983), but also allows both parties to have different probability beliefs about the insurance random loss. Under the assumption of general heterogeneous belief, a necessary and sufficient condition for the optimal ceded loss function is established. Then we discuss some interesting properties of the optimal solution then under this condition. Next, we discuss in detail the optimal problem of no insurance and full insurance which are of great significance in insurance economics, and give the specific numerical analysis in the case of no insurance, which supports our conclusion.

It is worth mentioning that our model is similar to the one of (Boonen, 2016). The difference is that (Boonen, 2016) takes minimizing the distorted risk mea- 
surement of the insured's risk exposure as the objective function, while we take maximizing the expected utility of the insured's final wealth as the objective function.

We also wish to point out that further research on this topic is needed. First, the research on one insurer can be extended to two or more insurers to establish the optimal solution. Secondly, we do not derive the explicit optimal solution for the general form of belief heterogeneity, but it's an interesting topic to consider some other ways to achieve the optimal strategy. We hope that these two important open problems can be addressed in future research.

\section{Acknowledgements}

The research was supported by Social Science Planning Project of Shandong Province (20CTJJ02).

\section{Conflicts of Interest}

The authors declare no conflicts of interest regarding the publication of this paper.

\section{References}

Arrow, K. J. (1963). Uncertainty and Welfare Economics of Medical Care. The American Economic Review, 53, 941-973. https://www.jstor.org/stable/1812044?seq=1

Boonen, T. J. (2016). Optimal Reinsurance with Heterogeneous Reference Probabilities. Risks, 4, 26. https://doi.org/10.3390/risks4030026

Cai, J., \& Tan, K. S. (2007). Optimal Retention for a Stop-Loss Reinsurance under the VaR and CTE Risk Measures. ASTIN Bulletin, 37, 93-112. https://doi.org/10.1017/S0515036100014756

Cheung, K. C. (2010). Optimal Reinsurance Revisited-A Geometric Approach. ASTIN Bulletin, 40, 221-239. https://doi.org/10.2143/AST.40.1.2049226

Chi, Y. (2019). On the Optimality of a Straight Deductible under Belief Heterogeneity. ASTIN Bulletin, 49, 242-263. https://doi.org/10.1017/asb.2018.30

Chi, Y., \& Lin, X. S. (2014). Optimal Reinsurance with Limited Ceded Risk: A Stochastic Dominance Approach. ASTIN Bulletin, 44, 103-126. https://doi.org/10.1017/asb.2013.28

Chi, Y., \& Zhuang, S. C. (2020). Optimal Insurance with Belief Heterogeneity and Incentive Compatibility. Insurance: Mathematics and Economics, 92, 104-114. https://doi.org/10.1016/j.insmatheco.2020.03.006

Ghossoub, M. (2016). Optimal Insurance with Heterogeneous Beliefs and Disagreement about Zero-Probability Events. Risks, 4, 29. https://doi.org/10.3390/risks4030029

Ghossoub, M. (2017). Arrow's Theorem of the Deductible with Heterogeneous Beliefs. North American Actuarial Journal, 21, 15-35. https://doi.org/10.1080/10920277.2016.1192477

Gollier, C. (2013). The Economics of Optimal Insurance Design. In G. Dionne (Ed.), Handbook of Insurance (2nd ed., pp. 107-122). New York: Springer. https://doi.org/10.1007/978-1-4614-0155-1_4

Huberman, G., Mayers, D., \& Smith Jr., C. W. (1983). Optimal Insurance Policy Indem- 
nity Schedules. The Bell Journal of Economics, 14, 415-426.

https://doi.org/10.2307/3003643

Jiang, W., Ren, J., Yang, C., \& Hong, H. (2019). On Optimal Reinsurance Treaties in Cooperative Game under Heterogeneous Beliefs. Insurance: Mathematics and Economics, 85, 173-184. https://doi.org/10.1016/j.insmatheco.2018.12.004

Liu, Z. B., Zhao, R. Q., Liu, X. Y., \& Chen, L. (2017). Contract Designing for a Supply Chain with Uncertain Information Based on Confidence Level. Applied Soft Computing, 56, 617-631. https://doi.org/10.1016/j.asoc.2016.05.054

Marshall, J. (1992). Optimum Insurance with Deviant Beliefs. In G. Dionne (Ed.), Contributions to Insurance Economics (pp. 255-274). Boston, MA: Kluwer Academic Publishers. https://doi.org/10.1007/978-94-017-1168-5_9

Savage, L. J. (1972). The Foundations of Statistics (2nd revised ed.). New York: Dover Publications, Inc.

Smith, V. L. (1968). Optimal Insurance Coverage. Journal of Political Economy, 76, 68-77. https://doi.org/10.1086/259382

Xu, Z. Q., Zhou, X. Y. \& Zhuang, S. C. (2019). Optimal Insurance with Rank-Dependent Utility and Incentive Compatibility. Mathematical Finance, 29, 659-692. https://doi.org/10.1111/mafi.12185

Young, V. R. (1999). Optimal Insurance under Wang's Premium Principle. Insurance: Mathematics and Economics, 25, 109-122.

https://doi.org/10.1016/S0167-6687(99)00012-8 